\title{
D1D5 microstate geometries from string amplitudes
}

\author{
Stefano Giusto, ${ }^{a}$ Jose F. Morales ${ }^{b}$ and Rodolfo Russo ${ }^{c}$ \\ ${ }^{a}$ Laboratoire de Physique Théorique et Hautes Energies, Université Pierre et Marie Curie - Paris 6, \\ 4 Place Jussieu, 75252 Paris cedex 05, France \\ ${ }^{b}$ INFN, Università di Roma "Tor Vergata", \\ Via delle ricerca scientifica 1, 00183, Roma, Italia \\ ${ }^{c}$ Centre for Research in String Theory, Department of Physics, Queen Mary University of London, \\ Mile End Road, London, E1 4NS, United Kingdom \\ E-mail: stefano.giusto@cea.fr, morales@roma2.infn.it, \\ r.russo@qmul.ac.uk
}

ABSTRACT: We reproduce the asymptotic expansion of the D1D5 microstate geometries by computing the emission amplitudes of closed string states from disks with mixed D1D5 boundary conditions. Thus we provide a direct link between the supergravity and D-brane descriptions of the D1D5 microstates at non-zero string coupling. Microscopically, the profile functions characterizing the microstate solutions are encoded in the choice of a condensate for the twisted open string states connecting D1 and D5 branes.

KEYwORDs: Black Holes in String Theory, D-branes

ARXiv EPRINT: 0912.2270 


\section{Contents}

1 Introduction 1

2 Review of D1D5 geometries 3

2.1 Asymptotic expansion of the geometry 5

$\begin{array}{lll}3 & \text { String vertex operators } & 6\end{array}$

3.1 Open string vertices 6

$\begin{array}{lll}3.2 & \text { Closed string vertex operators } & 9\end{array}$

4 Microstate geometries from disk amplitudes $\quad 10$

$\begin{array}{ll}4.1 \text { NSNS amplitude } & 11\end{array}$

$\begin{array}{lll}4.2 & \text { RR amplitude } & 13\end{array}$

5 Conclusions $\quad 15$

\section{Introduction}

The gravitational description of black hole microstates remains one of the fundamental and most debated problems, more than ten years after the seminal works $[1,2]$. While these works have shown that, at weak coupling, black hole microstates can be described and counted in terms of D-branes, their description when the gravitational coupling becomes finite is still considered an unsettled question. A possible answer to this question is provided by the "fuzzball proposal" (for reviews see [3-6]): in its essence, it states that the 'naive' geometry of black holes, in which the horizon is locally empty space, is modified by quantum gravity corrections up to scales of the horizon size. The proposal is motivated by the explicit construction of classical geometries with the same asymptotics than the black hole (or black string) solutions but with no horizon. The best understood example is that of the 2-charge BPS system in type IIB string theory, for which the geometries dual to the whole family of microstates has been constructed in [7-12]. The 2-charge system represents a somewhat degenerate example of black hole, in that its classical geometry has a singular horizon of zero size, and one needs higher derivative corrections to smooth out the singularity and produce a finite size horizon. It is thus of crucial importance to extend the construction of the geometries dual to microstates to the case of the 3-charge BPS black hole, which is likely to share the properties of general (extremal) black holes. Though many advances have been made towards this goal [13-19], a complete construction of 3-charge microstates is still missing. Part of the reason why this task has proved to be so challenging is that one lacks a systematic procedure to construct the geometry generated by a particular D-brane configuration.

To help close this gap, in this paper we provide the relation between the stringy description of the black hole microstates in terms of D-branes and the corresponding geometries of 
the gravitational description. In principle, once a D-brane configuration is fully understood at zero string coupling $\left(g_{s}=0\right)$, it is sufficient to switch on a non-zero coupling in order to derive the gravitational backreaction of the system. When $g_{s} \ll 1$ it is possible to use the conformal field theory description of D-branes to compute the corresponding gravitational profile perturbatively. In practice, one needs to compute disc amplitudes with the insertion of a closed string vertex: the boundary conditions on the disc should carry all the information of the D-brane configuration under analysis, while the closed string emitted represents the gravitational backreaction. For the half-BPS geometries the situation is quite simple and this mechanism has been checked explicitly in [20]. However this idea should work for any supergravity state that admits a description in terms of D-branes and, for instance, in [21] this approach was used to study a non-BPS configuration.

The situation we want to study is that of D-brane bound states where the single constituents of the system cannot be freely separated. The first non-trivial case is represented by the 2-charge systems in type IIB string theory. There are various dual descriptions of these configurations. For instance the geometries corresponding to each microstate of this system have been derived $[7,22,23]$ by using the description in terms of a fundamental string with a wave carrying a left (or right) moving momentum. These solutions are specified by a profile function, characterizing the profile of the fundamental string. Then by using a chain of dualities these geometries can be re-interpreted as solutions describing D1D5 bound states. Alternatively, these solutions can be derived by solving the $\frac{1}{4}$-BPS killing spinor equations of supergravity and can be thought as "bubblings" of the naive D1D5 geometry [24].

In this paper we address the study of the microstate geometries from the string theory perspective. In particular we will show how the leading asymptotics of the microstate geometries are reproduced by string amplitudes describing the emissions of closed string states from D1D5 disks. In principle we could start from the description of a D-string with a wave [25], derive the gravitational backreaction by using the boundary state for these D-branes [26] and then rewrite the result in the D1D5 duality frame. However, since our aim is to provide a direct link between the D-brane construction and the corresponding geometries, we will focus directly on the analysis of the D1D5 bound states. From the conformal field theory point of view these bound states are described by open string amplitudes with mixed boundary conditions. Mixed disks dual to the ones considered here were studied in [27], where a direct link between the open string emission and the gauge theory instantons was found. In this paper we will study the closed string emission from disks that have half of their boundary along the D1-branes and the other half along the D5-branes. We see that these simple amplitudes are sufficient to reproduce the first corrections that distinguish the microstate geometries from the naive superposition of D1 and D5-branes. These corrections display some of the fundamental properties of the fuzzball geometries, such as the fact that the non-trivial states carry some angular momentum that breaks the rotational invariance in the non-compact directions, and the presence of a Kaluza-Klein monopole dipole charge, which is ultimately responsible for the regularity of the geometries in their core. In the string description they can be associated to condensates of twisted open string states stretched between the D1 and D5 branes. The open string condensate, as we 
will see, encodes the information about the profile function characterizing the microstate supergravity solution.

The paper is organized as follows. In section 2 we review the solutions for the D1D5 microstate geometries of IIB supergravity on $\mathbb{R}^{4,1} \times S^{1} \times T^{4}$ and write explicitly the first order corrections to the naive D1D5 superposition. In section 3 we introduce all CFT ingredients we will need to compute the mixed disc amplitude mentioned above: we write the vertex operators for both the closed and the open strings, and discuss the identification between the holomorphic and antiholomorphic string coordinates induced by the D-branes. In section 4 we compute the emission of one closed string state from a D1D5 disk and show that they reproduce the long distance behavior of the microstate geometries reviewed in section 2. Finally, in the Conclusions, we discuss some possible generalisations and applications of our results.

\section{Review of D1D5 geometries}

Let us focus on type IIB string theory on $\mathbb{R}^{4,1} \times S^{1} \times T^{4}$. If one wraps $n_{1}$ D1 branes on $S^{1}$ and $n_{5}$ D5 branes on $S^{1} \times T^{4}$, one obtains a system that preserves $1 / 4$ of the 32 supersymmetries of type IIB strings and has, in the large $n_{1} n_{5}$ limit, $\exp \left(\sqrt{2} \pi \sqrt{n_{1} n_{5}}\right)$ states. The supergravity description of all these states was found in [7-12], exploiting the fact that the D1D5 system is U-dual to a fundamental string on $\mathbb{R}^{4,1} \times S^{\prime 1} \times T^{4}$ wrapped $n_{5}$ times on $S^{1}$ and carrying $n_{1}$ units of (left moving) momentum. The states of the fundamental string are described, in the semi-classical limit, by a curve in the space transverse to the string, $\mathbb{R}^{4} \times T^{\prime 4}$. We denote the parametric representation of this curve by $f_{A}(v)$, with $A=1, \ldots, 8, v=t-y$ and $y$ the coordinate on $S^{\prime 1}$ of radius $R^{\prime}$. The length of the multiply wound fundamental string is $L=2 \pi n_{5} R^{\prime}$, and the curve $f_{A}(v)$ is taken to have trivial winding along the $T^{4}$ directions, so that $f_{A}(v+L)=f_{A}(v)$, for any $A$. It will be convenient to distinguish the $\mathbb{R}^{4}$ directions, denoted by the indices $i, j, \cdots=1, \ldots, 4$, from the $T^{4}$ directions, labeled by $a, b, \ldots=5, \ldots, 8$. Moreover the U-duality connecting the D1D5 and the fundamental string descriptions of the system requires to pick a particular direction in the four dimensional torus. So the internal components $f_{a}(v)$ of the curve describing the fundamental string are not labeled by a vector index of the $T^{4}$ of the D1D5 description, but by an index $\hat{a}$ running over the three self-dual 2 -forms plus a scalar. In this notation the string profile is represented, in the D1D5 duality frame, by the functions

$$
f_{A}(v) \equiv\left(f_{i}(v), f_{\hat{a}}(v), f(v)\right) .
$$

We will choose the origin of our coordinates such as $\int_{0}^{L} f_{A}(v)=0$.

Along the time and the (compact) $y$ coordinate both the D1 and D5 branes have Neumann boundary conditions, while $x_{i}$ and $x_{a}$ parametrize $\mathbb{R}^{4}$ and $T^{4}$ where the D-branes have Dirichlet and mixed Neumann/Dirichlet boundary conditions respectively.

The string frame metric $\left(d s^{2}\right)$, dilaton $(\Phi)$, B-field $(b)$ and p-form RR fields $\left(C^{(p)}\right)$ of 
the generic D1D5 state are given by

$$
\begin{aligned}
d s^{2}= & \frac{\hat{H}_{1}^{1 / 2}}{\tilde{H}_{1} H_{5}^{1 / 2}}\left[-\left(d t-A_{i} d x_{i}\right)^{2}+\left(d y+B_{i} d x_{i}\right)^{2}\right]+\left(\hat{H}_{1} H_{5}\right)^{1 / 2} d x_{i} d x_{i}+\left(\frac{\hat{H}_{1}}{H_{5}}\right)^{1 / 2} d x_{a} d x_{a}, \\
e^{2 \Phi}= & \frac{\hat{H}_{1}^{2}}{\tilde{H}_{1} H_{5}}, \\
b= & -\frac{\mathcal{A}}{\tilde{H}_{1} H_{5}}(d t-A) \wedge(d y+B)+\mathcal{B}+\frac{\mathcal{A}_{\hat{a}} \omega^{\hat{a}}}{H_{5}}, \\
C^{(0)}= & -\frac{\mathcal{A}}{\hat{H}_{1}}, \\
C^{(2)}= & -d t \wedge d y+\tilde{H}_{1}^{-1}(d t-A) \wedge(d y+B)+C, \\
C^{(4)}= & -d t \wedge d y \wedge \mathcal{B}-\frac{\mathcal{A}}{H_{5} \tilde{H}_{1}}(d t-A) \wedge(d y+B) \wedge C-\frac{\mathcal{A}}{H_{5} \tilde{H}_{1}}(d t \wedge d y+C) \wedge A \wedge B \\
& -\frac{\mathcal{A}_{\hat{a}}}{H_{5}} d t \wedge d y \wedge \omega^{\hat{a}}+\left(\mathcal{B}_{\hat{a}}+\frac{\mathcal{A}_{\hat{a}}}{H_{5}} C\right) \wedge \omega^{\hat{a}}-\frac{\mathcal{A}}{H_{5}} d x_{5} \wedge d x_{6} \wedge d x_{7} \wedge d x_{8} .
\end{aligned}
$$

In our conventions the RR field strengths $F^{(p+1)}$ are defined as

$$
F^{(p+1)}=d C^{(p)}-H^{(3)} \wedge C^{(p-2)},
$$

with $H^{(3)}=d b$. The 5 -form field strength is taken to be self-dual ${ }^{1} F^{(5)}=* F^{(5)}$. The various functions appearing above are defined as follows

$$
\begin{aligned}
H_{5} & =1+\frac{Q_{5}}{L} \int_{0}^{L} \frac{d v}{\left|x_{i}-f_{i}(v)\right|^{2}}, & H_{1} & =1+\frac{Q_{5}}{L} \int_{0}^{L} \frac{d v\left|\dot{f}_{A}(v)\right|^{2}}{\left|x_{i}-f_{i}(v)\right|^{2}} \\
\hat{H}_{1} & =H_{1}-\frac{\mathcal{A}_{\hat{a}} \mathcal{A}_{\hat{a}}}{f_{5}}, & \tilde{H}_{1} & =H_{1}-\frac{\mathcal{A}_{\hat{a}} \mathcal{A}_{\hat{a}}+\mathcal{A} \mathcal{A}}{f_{5}} \\
A_{A} & =-\frac{Q_{5}}{L} \int_{0}^{L} \frac{d v \dot{f}_{A}(v)}{\left|x_{i}-f_{i}(v)\right|^{2}} \equiv\left(A_{i}, \mathcal{A}_{\hat{a}}, \mathcal{A}\right), & A & \equiv A_{i} d x_{i} \\
d B & =-*_{4} d A, \quad d C=-*_{4} d H_{5}, & d \mathcal{B}_{\hat{a}}=*_{4} d \mathcal{A}_{\hat{a}}, & d \mathcal{B}=*_{4} d \mathcal{A}
\end{aligned}
$$

where $*_{4}$ denotes the Hodge dual with respect to flat $\mathbb{R}^{4}$. Finally the three 2 -forms $\omega^{\hat{a}}$ form a basis for the self-dual forms $\star_{4} \omega^{\hat{a}}=\omega^{\hat{a}}$, where $\star_{4}$ is again defined with respect to a flat metric, but now acts on the indices in the $T^{4}$. The length $L$ can be expressed in terms of D1D5 quantities as

$$
L=2 \pi \frac{Q_{5}}{R}
$$

with $R$ the radius of $S^{1}$ in the D1D5 frame. The D1 charge is given by

$$
Q_{1}=\frac{Q_{5}}{L} \int_{0}^{L}\left|\dot{f}_{A}(v)\right|^{2}
$$

and the charges $Q_{1}$ and $Q_{5}$ are quantized as

$$
Q_{1}=\frac{(2 \pi)^{4} g \alpha^{\prime 3} n_{1}}{V_{4}}, \quad Q_{5}=g \alpha^{\prime} n_{5},
$$

with $V_{4}$ the volume of $T^{4}$ and $n_{1}, n_{5}$ the numbers of D1, D5 branes.

\footnotetext{
${ }^{1}$ Our conventions for the Hodge star are $(* a)_{i_{1} \ldots i_{d-p}}=\frac{1}{p !} \epsilon_{i_{1} \ldots i_{d-p} j_{1} \ldots j_{p}} a^{j_{1} \ldots j_{p}}$, with $\epsilon_{01 \ldots d}=\sqrt{|g|}$.
} 


\subsection{Asymptotic expansion of the geometry}

In the limit in which the backreaction of the D-branes on the geometry is small, the D1D5 system can be described by the perturbative dynamics of open strings stretched between the D-branes. This is the description we will focus on in the next section. This description should capture the large distance expansion of the geometries (2.2), which looks like a small perturbation around flat space.

We focus on the terms that distinguish the microstate geometries (2.2) from the naive D1D5 geometry, which is the singular, spherically symmetric, geometry with $f_{A}(v)=0$. These terms first appear at order $1 / r^{3}$ and are encoded in the functions $A_{i}, \mathcal{A}_{\hat{a}}, \mathcal{A}$ and in their duals $B_{i}, \mathcal{B}_{\hat{a} i j}, \mathcal{B}_{i j}$. Their large distance expansion is given by

$$
\begin{array}{ll}
A_{i} \approx-\frac{Q_{5}}{L} \int_{0}^{L} d v \dot{f}_{i}\left[\frac{1}{r^{2}}+2 \frac{x_{j} f_{j}}{r^{4}}\right]=-2 Q_{5} \hat{f}_{i j} \frac{x_{j}}{r^{4}}, \quad \hat{f}_{i j}=\frac{1}{L} \int_{0}^{L} d v \dot{f}_{i} f_{j}=-\hat{f}_{j i}, \\
\mathcal{A}_{\hat{a}} \approx-\frac{Q_{5}}{L} \int_{0}^{L} d v \dot{f}_{\hat{a}}\left[\frac{1}{r^{2}}+2 \frac{x_{j} f_{j}}{r^{4}}\right]=-2 Q_{5} \hat{f}_{\hat{a} j} \frac{x_{j}}{r^{4}}, \quad \hat{f}_{\hat{a} j}=\frac{1}{L} \int_{0}^{L} d v \dot{f}_{\hat{a}} f_{j}, \\
\mathcal{A} \approx-\frac{Q_{5}}{L} \int_{0}^{L} d v \dot{f}\left[\frac{1}{r^{2}}+2 \frac{x_{j} f_{j}}{r^{4}}\right]=-2 Q_{5} \hat{f}_{j} \frac{x_{j}}{r^{4}}, \quad \hat{f}_{j}=\frac{1}{L} \int_{0}^{L} d v \dot{f} f_{j},
\end{array}
$$

where we have used $\int_{0}^{L} \dot{f}_{A}=0$, and

$$
B_{i} \approx-Q_{5} \epsilon_{i j k l} \hat{f}_{k l} \frac{x_{j}}{r^{4}}, \quad \mathcal{B}_{\hat{a} i j} \approx-2 Q_{5} \epsilon_{i j k l} \hat{f}_{\hat{a} k} \frac{x_{l}}{r^{4}}, \quad \mathcal{B}_{i j} \approx-2 Q_{5} \epsilon_{i j k l} \hat{f}_{k} \frac{x_{l}}{r^{4}} .
$$

In this paper we consider D1D5 geometries that are invariant under the $\mathrm{SO}(4)$ acting along the ND directions (i.e. the $T^{4}$ coordinates). Hence we will focus on the solutions that have $\mathcal{A}_{\hat{a}}=\mathcal{B}_{\hat{a} i j}=0$. From (2.8)-(2.11) one finds that the asymptotic form of such solutions in the large distance limit is

$$
\begin{aligned}
g_{t i} & =-\frac{2 Q_{5} x_{j} \hat{f}_{i j}}{r^{4}}, & g_{y i} & =-\epsilon_{i j k l} \frac{Q_{5} x_{j} \hat{f}_{k l}}{r^{4}}, \\
b_{t y} & =\frac{2 Q_{5} x_{i} \hat{f}_{i}}{r^{4}}, & b_{i j} & =2 \epsilon_{i j k l} \frac{Q_{5} x_{k} \hat{f}_{l}}{r^{4}},
\end{aligned}
$$

for the NSNS fields and

$$
\begin{aligned}
C^{(0)} & =\frac{2 Q_{5} \hat{f}_{i} x_{i}}{r^{4}}, & C_{t i}^{(2)} & =-\epsilon_{i j k l} \frac{Q_{5} x_{j} \hat{f}_{k l}}{r^{4}}, \quad C_{y i}^{(2)}=-\frac{2 Q_{5} \hat{f}_{i j} x_{j}}{r^{4}}, \\
C_{\text {tyij }}^{(4)} & =-2 \epsilon_{i j k l} \frac{Q_{5} x_{k} \hat{f}_{l}}{r^{4}}, & C_{a b c d}^{(4)} & =\epsilon_{a b c d} \frac{2 Q_{5} \hat{f}_{i} x_{i}}{r^{4}},
\end{aligned}
$$

for the RR fields.

The simplest example of this type of configurations is provided by a circular profile in the plane 1,2

$$
f_{1}(v)=a \cos \frac{2 \pi w v}{L}, \quad f_{2}(v)=a \sin \frac{2 \pi w v}{L},
$$

while $f_{\hat{a}}, f$ and all remaining components of $f_{i}$ are zero. The eqs. (2.6) and (2.5) relate the amplitude $a$ of the profile to $Q_{1}$ and $Q_{5}$

$$
a=\frac{\sqrt{Q_{1} Q_{5}}}{w R} .
$$


In this case one can compute exactly all functions in (2.4), but for our purposes it is sufficient to look at the order captured by (2.8)-(2.10). By using (2.14) we find

$$
\hat{f}_{12}=-\hat{f}_{21}=-\frac{a^{2}}{2} \frac{2 \pi w}{L}=-\frac{Q_{1}}{2 w R}, \quad \hat{f}_{\hat{a} j}=\hat{f}_{j}=0 .
$$

By using this result in (2.12) and (2.13) and the quantization rule (2.7), one can see that all terms of order $1 / r^{3}$ are proportional to $n_{1} n_{5}$. This clearly suggests that the microscopic origin of these contributions is related to string diagrams involving both the D1 and the D5-branes at the same time.

\section{String vertex operators}

\subsection{Open string vertices}

A simple superposition of D1 and D5-branes does not represent a real bound state. From the conformal field theory point of view, this is signalled by the presence of massless string states describing the relative position of the two stacks of D-branes. We can lift these modes by giving a non-trivial vacuum expectation value to the open strings stretched between the two sets of D-branes. The effects of the condensate can be described in terms of string amplitudes with the insertions of twisted open string vertices. The insertion of a twisted open string state on the boundary of the disk flips a D1 boundary into a D5 and viceversa. From the supergravity solution the leading deviation from the naive D1D5 geometry arises at order $n_{1} n_{5}$ and therefore we need two twisted open string insertions.

There are two choices for the open string condensates depending on whether we excite states from the Neveu-Schwarz (NS) or the Ramond (R) sector of the open string theory. Turning a vacuum expectation value for the NS fields generates a non-trivial profile for the open string photons and can be interpreted as an instanton solution along the ND directions, see [27]. Here our setup is different in two respects: first the ND directions are compactified on a $T^{4}$, second we make a complementary choice for the open string condensate by turning on the states in the Ramond sector. As we will see, such a condensate does not generate any a non-trivial gauge (open string) profile, but only a supergravity (closed string) backreaction, reproducing the leading asymptotics of the microstate solutions. We restrict ourselves to systems with only two charges, (i.e. we set to zero the quantized momentum along the Neumann direction $S^{1}$, that would appear as an additional charge) and so we focus on the open string states at zero momentum.

We denote the 10D coordinates $\left(x^{\hat{M}}, \psi^{\hat{M}}\right)$ with $\hat{M}=t, y, 1, . .8$. It is convenient to parametrize the coordinates in terms of the light cone directions $Z^{ \pm}$and of four complex variables $Z^{I}$ :

$$
Z^{ \pm} \equiv \frac{1}{\sqrt{2}}(y \pm t), \quad Z^{n=1, . .4}=\frac{1}{\sqrt{2}}\left(x^{2 n-1}+\mathrm{i} x^{2 n}\right) .
$$

We will collectively denote the $\mathrm{NN}$ and DD directions by $Z^{I}, I=+,-, 1, \overline{1}, 2, \overline{2}$ and the mixed ND directions $Z^{a}, a=3, \overline{3}, 4, \overline{4}$. Indices $I, J, \ldots$ and $a, b, \ldots$ label the vector representations of the $\mathrm{SO}(1,5)$ and $\mathrm{SO}(4)$ Lorentz groups acting on the NN/DD and 
ND/DN planes respectively. In our conventions, the 10D Majorana-Weyl spinors $\Theta_{\hat{A}}$ satisfy $\Gamma_{(10)} \Theta_{\hat{A}}=-\Theta_{\hat{A}}$, where $\Gamma_{(10)}=\Gamma_{(10)}^{0} \Gamma_{(10)}^{y} \Gamma_{(10)}^{1} \ldots \Gamma_{(10)}^{8}$. These spinors decompose with respect to the $\mathrm{SO}(1,5) \times \mathrm{SO}(4)$ as

$$
\Theta_{\hat{A}}=\left\{\Theta_{A}^{\dot{\alpha}} ; \Theta^{A \alpha}\right\}
$$

where upper and lower indices $A, B, \cdots=1, \ldots, 4$ denote Weyl $\mathrm{SO}(1,5)$ spinors of opposite chirality; similarly $\alpha, \dot{\alpha}=1,2$ are Weyl spinor indices of opposite chirality for the $\mathrm{SO}(4)$ group acting along the ND $T^{4}$ directions. We decompose the 10D Gamma matrices as follows

$$
\Gamma_{(10)}^{a}=1_{(6)} \otimes \gamma^{a}, \quad \Gamma_{(10)}^{I}=\Gamma^{I} \otimes \gamma^{\mathrm{ND}},
$$

where we use simply $\Gamma^{I}$ for the $6 \mathrm{D}$ Gamma matrices and

$$
\begin{aligned}
\left(\gamma^{\mathrm{ND}}\right)_{\dot{\alpha}}^{\dot{\beta}} & =\left(\prod_{a} \gamma^{a}\right)_{\dot{\alpha}}^{\dot{\beta}}=-\delta_{\dot{\alpha}}^{\dot{\beta}}, \quad\left(\gamma^{\mathrm{ND}}\right)_{\alpha}^{\beta}=\left(\prod_{a} \gamma^{a}\right)_{\alpha}^{\beta}=\delta_{\alpha}^{\beta}, \\
(\Gamma)_{A}^{B} & =\left(\prod_{I} \Gamma^{I}\right)_{A}^{B}=-\delta_{A}^{B}, \quad(\Gamma)_{B}^{A}=\left(\prod_{I} \Gamma^{I}\right)_{B}^{A}=\delta_{B}^{A} .
\end{aligned}
$$

Instead of the 6D Gamma matrices, we will often use the chiral components such as $\left(C \Gamma^{I_{1} . . I_{2 n-1}}\right)_{A B}$, where $C$ is the $6 \mathrm{D}$ charge conjugation matrix ${ }^{2}$ satisfying ${ }^{\mathrm{t}} \Gamma^{I}=-C \Gamma^{I} C^{-1}$.

We will consider string amplitudes describing a closed string emission from a mixed D1D5 disk with twisted open string vertex insertions. We refer the reader to [28] for a general discussion of closed string amplitudes on disks with mixed boundary conditions and for details on the conventions we follow here. Twisted open string vertices are associated to string states stretched between D-branes with different boundary conditions and involve bosonic twist fields. Since there is no simple conformal field theory description when these fields have a non-zero expectation value, we will work perturbatively, and consider the leading contribution coming from the insertion of a single pair of twisted open string vertices. The open string vertices flip the boundary conditions on the disk from D1 to D5 types and viceversa and therefore split the disk boundary into two portions with D1 and D5 boundary conditions respectively. We restrict ourselves to massless physical states describing the lowest excitations of open strings stretched between D1 and D5 branes (for more details see, for example, [27]). In the NS sector, these states are generated by the zero-modes of the $T^{4}$ fermions, $\psi^{a}$, and hence form a spinor representation of $\mathrm{SO}(4)$. The associated vertex operators are

$$
V_{w}=w_{\dot{\alpha}} \mathrm{e}^{-\varphi} S^{\dot{\alpha}} \Delta, \quad V_{\bar{w}}=\bar{w}_{\dot{\alpha}} \mathrm{e}^{-\varphi} S^{\dot{\alpha}} \Delta
$$

with $w_{\dot{\alpha}}, \bar{w}_{\dot{\alpha}}$ denoting $n_{1} \times n_{5}$ and $n_{5} \times n_{1}$ Chan-Paton matrices respectively. In the $\mathrm{R}$ sector, it is the fermions along the $I$ directions that have zero modes, and hence the physical states form a spinor representation of $\mathrm{SO}(1,5)$. They correspond to the vertex operators

$$
V_{\mu}=\mu^{A} \mathrm{e}^{-\frac{\varphi}{2}} S_{A} \Delta, \quad V_{\bar{\mu}}=\bar{\mu}^{A} \mathrm{e}^{-\frac{\varphi}{2}} S_{A} \Delta,
$$

\footnotetext{
${ }^{2} C$ is related to the $10 \mathrm{D}$ and $4 \mathrm{D}$ charge conjugation matrices by $C_{10}=C \otimes C_{4}$.
} 
where the Chan-Paton matrices $\mu^{A}$ and $\bar{\mu}^{A}$ have $n_{1} \times n_{5}$ and $n_{5} \times n_{1}$ components respectively. In (3.5), (3.6) and below, we denote by $\varphi$ the free boson appearing in the bosonized language of the worldsheet superghost $(\beta, \gamma) . \Delta$ is the bosonic twist operator with conformal dimension $\frac{1}{4}$, that acts along the four mixed ND directions and changes the boundary conditions from Neumann to Dirichlet and viceversa. $S^{\alpha}$ and $S_{A}$ are the $\mathrm{SO}(4)$ and $\mathrm{SO}(1,5)$ spin fields. After bosonization these spin fields are simply exponentials of free bosons. If one introduces the bosons $h_{\mathbf{I}}=\left(h_{+}, h_{1}, h_{2}\right)$ and $h_{\mathbf{a}}=\left(h_{3}, h_{4}\right)$ associated to the $\mathrm{SO}(1,5)$ and $\mathrm{SO}(4)$ fermions respectively, fermions and spin fields are given by ${ }^{3}$

$$
\begin{aligned}
& S^{A}=S^{\vec{\epsilon}^{A}}=\mathrm{e}^{\frac{\mathrm{i}}{2} \epsilon^{A \mathbf{I}} h_{\mathbf{I}}}, \quad S_{A}=S^{\vec{\epsilon}_{A}}=\mathrm{e}^{\frac{\mathrm{i}}{2} \epsilon_{A}^{\mathbf{I}} h_{\mathbf{I}}}, \quad \psi^{\mathbf{I}}=\mathrm{e}^{\mathrm{i} h_{\mathbf{I}}}, \quad \bar{\psi}^{\mathbf{I}}=\mathrm{e}^{-\mathrm{i} h_{\mathbf{I}}}, \\
& S^{\dot{\alpha}}=S^{\vec{\epsilon}_{\dot{\alpha}}}=\mathrm{e}^{\frac{i}{2} \epsilon_{\dot{\alpha}}^{\mathbf{a}} h_{\mathbf{a}}}, \quad S^{\alpha}=S^{\vec{\epsilon}_{\alpha}}=\mathrm{e}^{\frac{\mathrm{i}}{2} \epsilon_{\alpha}^{\mathbf{a}} h_{\mathbf{a}}}, \quad \psi^{\mathbf{a}}=\mathrm{e}^{\mathrm{i} h_{\mathbf{a}}}, \quad \bar{\psi}^{\mathbf{a}}=\mathrm{e}^{-\mathrm{i} h_{\mathbf{a}}},
\end{aligned}
$$

with $\mathbf{I}=+, 1,2, \mathbf{a}=1,2$ running over the holomorphic components and $A, \alpha, \dot{\alpha}$ running over the spinor components corresponding to the following choices of signs

$$
\begin{aligned}
& \vec{\epsilon}_{A}=\{(---),(-++),(+-+),(++-)\}, \\
& \vec{\epsilon}^{A}=\{(+++),(+--),(-+-),(--+)\}, \\
& \vec{\epsilon}_{\alpha}=\{(++),(--)\}, \\
& \vec{\epsilon}_{\dot{\alpha}}=\{(+-),(-+)\} .
\end{aligned}
$$

As we mentioned before, we focus on open string condensates involving only states from the Ramond sector. Notice that states in the Ramond sector will break the SO(4) symmetry of the DD directions $\mathbb{R}^{4}$, while they are invariant under the $\mathrm{SO}(4)$ acting on the compact $T^{4}$ torus. They will be then associated with those supergravity solutions in (2.2) with $\mathcal{A}_{\hat{a}}=0$. The most general condensate of Ramond open strings can be written as:

$$
\bar{\mu}^{A} \mu^{B}=v_{I}\left(C \Gamma^{I}\right)^{[A B]}+\frac{1}{3 !} v_{I J K}\left(C \Gamma^{I J K}\right)^{(A B)},
$$

where the parenthesis on the indices $A, B$ are meant to remind that the first term is automatically antisymmetric, while the second one is symmetric. Thus the open string bispinor condensate is specified by a one-form $v_{I}$ and an self-dual three-form $v_{I J K}$. The self-duality of $v_{I J K}$ follows from $\bar{\mu}^{A}$ and $\mu^{B}$ having definite $6 \mathrm{D}$ chirality and can be written as

$$
v_{I J K}=\frac{1}{3 !} \epsilon_{I J K L M N} v^{L M N} .
$$

We will focus on symmetric open string condensates satisfying $v_{I}=0$. In fact, turning on a vacuum expectation value for $v_{I}$ would generate a tadpole for the D1D1 and D5D5 untwisted fields ${ }^{4} A_{I}^{(1)}, A_{I}^{(5)}$. This is not the case of $v_{I J K}$ components that do not have any trilinear coupling with open string states and therefore one can turn on a condensate $\left\langle v_{I J K}\right\rangle \neq 0$ without generating a tadpole in the open string theory. We will consider D1D5 geometries generated by the mixed disks involving these non-trivial condensates, i.e.

$$
\bar{\mu}^{(A} \mu^{B)}=\frac{1}{3 !} v_{I J K}\left(C \Gamma^{I J K}\right)^{A B}, \quad v_{I J K}=-\frac{1}{8} \bar{\mu}^{A}\left(\Gamma_{I J K} C^{-1}\right)_{A B} \mu^{B} .
$$

\footnotetext{
${ }^{3}$ In our conventions $h_{\mathbf{I}}\left(z_{1}\right) h_{\mathbf{J}}\left(z_{2}\right) \sim-\delta_{\mathbf{I} \mathbf{J}} \log z_{12}, h_{\mathbf{a}}\left(z_{1}\right) h_{\mathbf{b}}\left(z_{2}\right) \sim-\delta_{\mathbf{a b}} \log z_{12} . h_{\mathbf{I} \neq+}$ are real fields and $h_{+}$purely imaginary [29].

${ }^{4}$ This can be seen by computing the three point function $\left\langle V_{\mu} V_{A} V_{\bar{\mu}}\right\rangle$ with $V_{A}=e^{-\varphi} \psi^{I}$.
} 
It has to be remembered that the spinors $\bar{\mu}^{A}$ and $\mu^{B}$ carry $n_{5} \times n_{1}$ and $n_{1} \times n_{5}$ Chan-Paton indices, and hence the condensate $\bar{\mu}^{A} \mu^{B}$ has to be thought of as the vev for the sum

$$
\sum_{m=1}^{n_{1}} \sum_{n=1}^{n_{5}} \bar{\mu}_{m n}^{A} \mu_{n m}^{B}
$$

which, for generic choices of the Chan-Paton factors, is of order $n_{1} n_{5}$. We thus see that the amplitudes we compute are of the same order in $n_{1} n_{5}$ as the gravity terms (2.12) and (2.13). In the following we will assume that the second identity in (3.11) already contains the trace over Chan-Paton indices, and thus that $v_{I J K}$ is of order $n_{1} n_{5}$.

\subsection{Closed string vertex operators}

The vertex operators in the closed string sector $\operatorname{are}^{5}$

$$
\begin{aligned}
W_{\mathrm{NS}} & =\mathcal{G}_{\hat{M} \hat{N}}\left(\partial X_{L}^{\hat{M}}-\mathrm{i} k_{L} \cdot \psi \psi^{\hat{M}}\right) \mathrm{e}^{\mathrm{i} k_{L} X_{L}}(z) \tilde{\psi}^{\hat{N}} \mathrm{e}^{-\tilde{\varphi}} \mathrm{e}^{\mathrm{i} k_{R} X_{R}}(\bar{z}), \\
W_{\mathrm{R}} & =\frac{1}{4 \sqrt{2}} \mathcal{F}_{\hat{A} \hat{B}} \mathrm{e}^{-\frac{\varphi}{2}} S^{\hat{A}} \mathrm{e}^{\mathrm{i} k_{L} X_{L}}(z) \mathrm{e}^{-\frac{\tilde{\varphi}}{2}} \tilde{S}^{\hat{B}} \mathrm{e}^{\mathrm{i} k_{R} X_{R}}(\bar{z})
\end{aligned}
$$

where all hatted indices are ten dimensional and $\mathcal{G}_{\hat{M} \hat{N}}$ contains the $10 \mathrm{D}$ metric, the NSNS 2-form, and the dilaton, while $\mathcal{F}_{\hat{A} \hat{B}}$ is a RR field strength which can be expanded on a basis of ten dimensional Gamma matrices and contains a 1, a 3 and a self-dual 5-form

$$
\mathcal{F}_{\hat{A} \hat{B}}=\sum_{n=1,3,5} \frac{1}{n !} F_{\hat{M}_{1} \ldots \hat{M}_{n}}^{(n)}\left(C_{10} \Gamma_{(10)}^{\hat{M}_{1} \ldots \hat{M}_{n}}\right)_{\hat{A} \hat{B}} .
$$

Closed string vertices are separately normal ordered in the left and right moving terms. This is important in disk amplitudes where the left and right moving fields are identified

$$
\tilde{\varphi}=\varphi \quad, \quad X_{R}^{\hat{M}}=R_{\hat{N}}^{\hat{M}} X_{L}^{\hat{N}} \quad, \quad \tilde{\psi}^{\hat{M}}=R_{\hat{N}}^{\hat{M}} \psi^{\hat{N}} \quad, \quad \tilde{S}^{\hat{A}}=R_{\hat{B}}^{\hat{A}} S^{\hat{B}} .
$$

The identification matrix $R$ depends on the D-brane boundary conditions: $R_{\hat{N}}^{\hat{M}}$ is a diagonal matrix with -1 along the Dirichlet directions and +1 otherwise, while the identification matrices with spinor indices is, up to a sign, the product of the (ten dimensional) Gamma matrices along the Neumann directions. In our case, we have two possible choices for $R$, depending whether we use the boundary conditions of the D1 or the D5 branes

$$
R_{\mathrm{D} 1}=\Gamma_{(10)}^{t y}=\Gamma_{(10)}^{+-}, \quad R_{\mathrm{D} 5}=\Gamma_{(10)}^{t y 5678}=-\Gamma_{(10)}^{+-3 \overline{3} 4 \overline{4}}
$$

with $\Gamma_{(10)}^{5678}$ the chirality operator along $T^{4}$ written in terms of the 10D Gamma matrices. The final result of each correlator should not depend on the particular choice made for $R$. Here we will always take $R=R_{\mathrm{D}_{1}}$.

As we are interested in configurations that are translationally invariant both along the world sheet directions $t$ and $y$ and the $T^{4}$ directions, we take the closed string momentum

\footnotetext{
${ }^{5}$ For our purposes we will not need the absolute normalization of the vertex operators; the relative normalization is determined by requiring that the NSNS and RR states are related to the canonically normalized fields with the same proportionality constant (for details see [30]).
} 
vector along $\mathbb{R}^{4}: k_{L}^{i}=k_{R}^{i}=k^{i} / 2$. The asymptotics of the D1D5 geometries generated by the mixed disks will be captured by the leading term in the expansion of the string amplitude for small $k_{i}$. We are interested only on the leading term in the momentum expansion, and therefore we can set to zero the momentum in the exponential of closed string vertices. Finally, since the open string condensate $\bar{\mu}^{(A} \mu^{B)}$ under consideration is invariant under the $\mathrm{SO}(4)$ Lorentz group of the $T^{4}$ torus, we can restrict ourselves to $\mathrm{SO}(4)$ invariant components $\mathcal{G}_{I J}, \mathcal{F}_{A B[\dot{\alpha} \dot{\beta}]}, \mathcal{F}^{A B[\alpha \beta]}$ in (3.13) and (3.14). In addition the RR components $\mathcal{F}^{A B[\alpha \beta]}$ can be discarded by noticing that the only $\mathrm{SO}(6)$ singlet $\epsilon_{A B C D} \mu^{A} \bar{\mu}^{B} \mathcal{F}^{C D}[\alpha \beta]$ vanishes for the symmetric open string condensate $\bar{\mu}^{(A} \mu^{B)}$ considered here.

\section{Microstate geometries from disk amplitudes}

In this section we show how the small $g_{s}$, long distance behavior of the microstate solutions (2.2) is reproduced by using string amplitudes and the microscopic description of D-branes. In particular, we compute the emission of one closed string state from various mixed disks (i.e. disks that have half of their boundary along the D1-branes and the other half along the D5-branes) and extract from these amplitudes the leading deviation of the geometries (2.2) from the naive D1D5 metric, which appears at order $1 / r^{3}$ in the large $r$ limit. We will restrict ourselves to open string condensates describing D1D5 geometries with trivial profile along the compact $T^{4}$ directions, i.e. $\mathcal{A}_{\hat{a}}=\mathcal{B}_{\hat{a} i j}=0$. The correlators we compute reproduce the terms in the geometries (2.2) of order $1 / r^{3}$, which are captured by the functions $A_{i}, \mathcal{A}$, and their duals $B_{i}, \mathcal{B}_{i j}$ given in eqs. (2.8)-(2.11).

The emission of closed string states from a D1D5 system with an open string condensate turned on can be described by computing string diagrams with a closed string state and some number of open string insertions. The leading contribution comes from the disk with one closed and two twisted open string vertices. The insertion of two twisted open string vertices divides the disk into two boundaries with D1 and D5 boundary conditions. The relevant correlator can be written as

$$
\mathcal{A}_{\mathrm{W}}=\int \frac{\prod_{i=1}^{4} d z_{i}}{d V_{\mathrm{CKG}}}\left\langle V_{\mu}\left(z_{1}\right) W_{\mathrm{NS}, \mathrm{R}}\left(z_{2}, z_{3}\right) V_{\bar{\mu}}\left(z_{4}\right)\right\rangle
$$

where $W_{\mathrm{NS}, \mathrm{R}}$ represents the emitted closed string. The closed string operators to be inserted in (4.1) need to have total superghost charge -1 so that it compensates, together with the superghost charge of the open string vertices, the -2 background charge of the disk. The relevant vertex operators are given by (3.6), (3.13), (3.14). The open string variables, denoted by $z_{1}$ and $z_{4}$, are integrated on the real axis while the closed string variables $z_{2}=z$ and $z_{3}=\bar{z}$ are the complex conjugate of each other and $z$ must be integrated over the upper half complex plane. The $\mathrm{SL}(2 ; \mathbb{R})$ projective invariance is fixed explicitly by choosing

$$
\frac{\prod_{i=1}^{4} d z_{i}}{d V_{\mathrm{CKG}}}=d \omega\left(z_{13} z_{24}\right)^{2}
$$


where $\omega$ is the ratio ${ }^{6}$

$$
\omega=\frac{z_{12} z_{34}}{z_{13} z_{24}}
$$

with $z_{i j}=z_{i}-z_{j}$. Notice that $\omega$ is a pure phase since $z_{2}$ and $z_{3}$ are complex conjugate of each other, while $z_{1}$ and $z_{4}$ are real.

\subsection{NSNS amplitude}

Let us start from the emission of the NSNS state

$$
\mathcal{A}_{\mathrm{NS}}=\int \frac{\prod_{i=1}^{4} d z_{i}}{d V_{\mathrm{CKG}}}\left\langle V_{\mu}\left(z_{1}\right) W_{\mathrm{NS}}\left(z_{2}, z_{3}\right) V_{\bar{\mu}}\left(z_{4}\right)\right\rangle .
$$

The transversality condition $k^{I} \mathcal{G}_{I J}=0$ implies that the term of the closed vertex (3.13) proportional to $\partial X^{\hat{M}}$ does not contribute to the correlator: only the term proportional to $k_{L} \cdot \psi \psi^{\hat{M}}$ contributes. Only one Lorentz invariant can be built out of $k_{K},(\mathcal{G} R)_{I J}$ and $v_{I J K}$ and so the form of the string amplitude is determined from Lorentz invariance up to a constant $\mathcal{I}$

$$
\mathcal{A}_{\mathrm{NS}}=-k_{K}(\mathcal{G} R)_{I J} \bar{\mu}^{A} \mu^{B}\left(\Gamma^{I J K} C^{-1}\right)_{A B} \mathcal{I}=8 k_{K}(\mathcal{G} R)_{I J} v^{I J K} \mathcal{I},
$$

where the last equality follows from the second relation in (3.11). It is important to stress that the CFT correlator is $\mathrm{SO}(1,5)$ invariant, and only the explicit form of the matrix $R$ breaks this invariance down to $\mathrm{SO}(1,1) \times \mathrm{SO}(4)$. The constant $\mathcal{I}$ has to be computed from the explicit evaluation of the CFT correlator. To evaluate $\mathcal{I}$, we specify to the term in (4.5) with $K=1, I=2, J=+$ and $\vec{\epsilon}_{A}=\vec{\epsilon}_{B}=(---)$. Notice that for this choice of open string polarizations, the indices $A, B$ are automatically symmetric consistently with the desired form of the $\bar{\mu} \mu$-condensate (see eq. (3.11)). Charge conservation implies that only the cubic fermionic term of the vertex (3.13), in which all the three fermionic fields carry $\mathrm{SO}(1,5)$ indices, can contribute to this amplitude and therefore one can write

$$
W_{\mathrm{NS}}=-\mathrm{i} \frac{k_{K}}{2}(\mathcal{G} R)_{I J} \psi^{K} \psi^{I}\left(z_{2}\right) \psi^{J}\left(z_{3}\right) .
$$

Thus the relevant correlators are

$$
\begin{aligned}
\left\langle\Delta\left(z_{1}\right) \Delta\left(z_{4}\right)\right\rangle & =z_{14}^{-\frac{1}{2}} \\
\left\langle\mathrm{e}^{-\frac{\varphi}{2}}\left(z_{1}\right) \mathrm{e}^{-\varphi}\left(z_{3}\right) \mathrm{e}^{-\frac{\varphi}{2}}\left(z_{4}\right)\right\rangle & =\left(z_{13} z_{34}\right)^{-\frac{1}{2}} z_{14}^{-\frac{1}{4}}, \\
\left\langle S^{---}\left(z_{1}\right) \psi^{1} \psi^{2}\left(z_{2}\right) \psi^{+}\left(z_{3}\right) S^{---}\left(z_{4}\right)\right\rangle & =\left(z_{12} z_{24}\right)^{-1}\left(z_{13} z_{34}\right)^{-\frac{1}{2}} z_{14}^{\frac{3}{4}} .
\end{aligned}
$$

The last equation can be written in the covariant form ${ }^{7}$

$$
\left\langle S_{(A}\left(z_{1}\right) \psi^{I} \psi^{J}\left(z_{2}\right) \psi^{K}\left(z_{3}\right) S_{B)}\left(z_{4}\right)\right\rangle=\frac{1}{2 \sqrt{2}}\left(\Gamma^{I J K} C^{-1}\right)_{A B}\left(z_{12} z_{24}\right)^{-1}\left(z_{13} z_{34}\right)^{-\frac{1}{2}} z_{14}^{\frac{3}{4}} .
$$

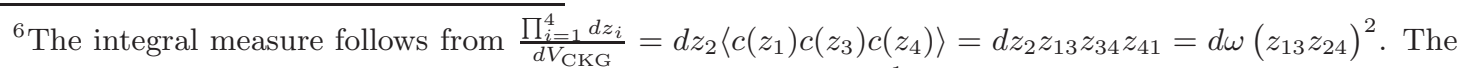
other two ratios that can be built from $\omega$ are $1-\omega=\frac{z_{23} z_{14}}{z_{13} z_{24}}$ and $\frac{1-\omega}{\omega}=\frac{z_{23} z_{14}}{z_{12} z_{34}}$.

${ }^{7}$ The origin of the factors of $\sqrt{2}$ lies in the OPE's of the $\psi^{I}$ 's and the twist fields [27, 31]:
}

$$
\psi^{I}(z) S_{A}(0) \sim \frac{1}{\sqrt{2}} \frac{\Gamma_{A B}^{I} S^{B}(0)}{z^{1 / 2}} .
$$


By inserting eqs. (4.2), (4.7), (4.8) into (4.4), one finds

$$
\mathcal{I}=\frac{\mathrm{i}}{4 \sqrt{2}} \int \frac{d \omega}{\omega}=-\frac{\pi}{2 \sqrt{2}}
$$

with the integral running over the unitary circle. Substituting the value of $\mathcal{I}$ in (4.5), one finds that the string amplitude is

$$
\mathcal{A}_{\mathrm{NS}}=-2 \sqrt{2} \pi k_{K}(\mathcal{G} R)_{I J} v^{I J K} .
$$

As we will now show, this amplitude exactly reproduces the $1 / r^{3}$ contributions to the metric and the B-field in (2.2) associated with functions $A_{i}, B_{i}, \mathcal{A}$ and $\mathcal{B}$. In order to identify the fields appearing in (4.10), we need to decompose the $\mathrm{SO}(1,5)$ vector indices into $\mathrm{SO}(1,1) \times \mathrm{SO}(4)$ indices $\left(I=(t, y, i)\right.$, where, as before, $i=1, . .4$ label the $\mathbb{R}^{4}$ Dirichlet directions and $t, y$ the Neumann directions). Notice that only antisymmetric components $(\mathcal{G R})_{[J K]}$ contribute to the amplitude. Using the fact that the matrix $R$ is +1 along $t, y$ and -1 otherwise, one finds that the matrix $(\mathcal{G R})_{J K}$ is antisymmetric if and only if $\mathcal{G}$ is antisymmetric (B-field) and the $(J, K)$ indices are of the same type (i.e. $(t, y)$ or $(i, j))$, or $\mathcal{G}$ is symmetric (metric) and the $(J, K)$ indices are of the different type (i.e. $(t, i)$ or $(y, i))$. This implies that only the components $g_{t i}, g_{y i}$ and $b_{t y}, b_{k l}$ are emitted from the mixed disk. In addition we recall that the momentum $k_{I}$ of the closed string is non-zero only in the Dirichlet directions $i$. The amplitude (4.10) can then be written as

$$
\begin{aligned}
\mathcal{A}_{\mathrm{NS}} & =4 \sqrt{2} \pi\left(k_{j} g_{t i} v^{t i j}+k_{j} g_{y i} v^{y i j}-k_{i} b_{t y} v^{t y i}+\frac{1}{2} k_{k} b_{i j} v^{i j k}\right) \\
& =4 \sqrt{2} \pi\left(k^{j} g^{t i} v_{t i j}+\frac{1}{2} k^{j} g^{y i} \epsilon_{i j k l} v_{t k l}-k^{i} b^{t y} v_{t y i}-\frac{1}{2} k^{k} b^{i j} \epsilon_{i j k l} v_{t y l}\right),
\end{aligned}
$$

where in the second line we used the self-duality conditions of the 3 -form $v^{I J K}$ given by

$$
v_{y i j}=\frac{1}{2} \epsilon_{i j k l} v_{t k l} \quad v_{i j k}=-\epsilon_{i j k l} v_{t y l} .
$$

From (4.11) we can read the profile of the induced metric and $B$ field, for instance

$$
g_{t i}(k)=\frac{1}{2} \frac{\delta \mathcal{A}_{\mathrm{NS}}}{\delta g^{t i}}=2 \sqrt{2} \pi k_{j} v_{t i j}, \quad b_{t y}(k)=\frac{\delta \mathcal{A}_{\mathrm{NS}}}{\delta b^{t y}}=-4 \sqrt{2} \pi k_{j} v_{t y j} .
$$

As in [20], the space-time configuration associated with a closed string emission amplitude is obtained by multiplying the derivative of the amplitude with respect to the closed string field by a free propagator and taking the Fourier transform. In general for a field $a_{\mu_{1} \ldots \mu_{n}}$ we have

$$
a_{\mu_{1} \ldots \mu_{n}}(x)=\int \frac{d^{4} k}{(2 \pi)^{4}}\left(-\frac{\mathrm{i}}{k^{2}}\right) a_{\mu_{1} \ldots \mu_{n}}(k) \mathrm{e}^{\mathrm{i} k x},
$$

Note that that equation (4.8) is verified also for other choices of the polarizations $\vec{\epsilon}_{A, B}$. Take, for example, $\vec{\epsilon}_{A}=(---), \vec{\epsilon}_{B}=(-++)$ and $I=+, J=1, K=\overline{1}$. Then on one side one has

$$
\left\langle S^{(---}\left(z_{1}\right) \psi^{+} \psi^{1}\left(z_{2}\right) \psi^{\overline{1}}\left(z_{3}\right) S^{-++)}\left(z_{4}\right)\right\rangle=\frac{1}{2}\left(z_{12} z_{24}\right)^{-1}\left(z_{13} z_{34}\right)^{-\frac{1}{2}} z_{14}^{\frac{3}{4}},
$$

and on the other side

$$
\left(\Gamma^{+1 \overline{1}}\right)_{---,-++}=\frac{1}{2}\left(\Gamma^{+} \Gamma^{1} \Gamma^{\overline{1}}\right)_{---,-++}=\sqrt{2},
$$

which agrees with (4.8). 
with $a_{\mu_{1} \ldots \mu_{n}}(k)$ given in terms of derivatives of $\mathcal{A}$ as in (4.13). In our case, the Fourier transform has the following form

$$
\int \frac{d^{4} k}{(2 \pi)^{4}}\left(-\frac{\mathrm{i}}{k^{2}}\right) k_{j} \mathrm{e} \mathrm{e}^{\mathrm{i} k x}=-\frac{\partial}{\partial x^{j}} \int \frac{d^{4} k}{(2 \pi)^{4}} \frac{1}{k^{2}} \mathrm{e}^{\mathrm{i} k x}=-\frac{1}{2 \pi^{2}} \frac{x_{j}}{r^{4}},
$$

where we used

$$
\int \frac{d^{4} k}{(2 \pi)^{4}} \frac{e^{\mathrm{i} k x}}{k^{2}}=-\frac{1}{4 \pi^{2}} \frac{1}{r^{2}} .
$$

Thus from (4.13) we get the following results for the large distance behavior of $g_{t i}$ and $b_{t y}$ at order $1 / r^{3}$

$$
g_{t i}(x)=-\frac{\sqrt{2}}{\pi} \frac{x^{j} v_{t i j}}{r^{4}}, \quad b_{t y}(x)=\frac{2 \sqrt{2}}{\pi} \frac{x^{i} v_{t y i}}{r^{4}} .
$$

We can follow the same steps for the variation of $\mathcal{A}_{\mathrm{NS}}$ in (4.11) with the respect to $g_{y i}$ and $b_{i j}$ leading to

$$
g_{y i}(x)=-\frac{1}{\sqrt{2} \pi} \epsilon_{i j k l} \frac{x^{j} v_{t k l}}{r^{4}}, \quad b_{i j}(x)=\frac{2 \sqrt{2}}{\pi} \epsilon_{i j k l} \frac{x^{k} v_{t y l}}{r^{4}} .
$$

Comparing the values of $g_{t i}$ and $b_{t y}$ derived above with the $1 / r^{3}$ terms of the gravity solution (2.12), one fixes the identification between the string condensate parameter $v_{I J K}$ and the parameters $\hat{f}_{i j}$ and $\hat{f}_{i}$ that characterize the gravity solution at this order:

$$
Q_{5} \hat{f}_{i j}=\frac{1}{\sqrt{2} \pi} v_{t i j}, \quad Q_{5} \hat{f}_{i}=\frac{\sqrt{2}}{\pi} v_{t y i} .
$$

Using this identification, one verifies that the components $g_{y i}$ and $b_{i j}$ predicted by the string computation agree with the gravity result (2.12).

\subsection{RR amplitude}

Let us now consider the amplitude describing the emission of RR states

$$
\mathcal{A}_{\mathrm{R}}=\int \frac{\prod_{i=1}^{4} d z_{i}}{d V_{\mathrm{CKG}}}\left\langle V_{\mu}\left(z_{1}\right) W_{\mathrm{R}}\left(z_{2}, z_{3}\right) V_{\bar{\mu}}\left(z_{4}\right)\right\rangle
$$

with $W_{\mathrm{R}}$ given by (3.14). As discussed at the end of section 3, the open string condensate under analysis contributes only to the emission of

$$
W_{\mathrm{R}}^{\mathrm{ef}}=\frac{1}{4 \sqrt{2}}(\mathcal{F} R)_{A B} \epsilon_{\dot{\alpha} \dot{\beta}} \mathrm{e}^{-\frac{\varphi}{2}} S^{A} S^{\dot{\alpha}}(z) \mathrm{e}^{-\frac{\varphi}{2}} S^{B} S^{\dot{\beta}}(\bar{z}),
$$

with

$$
\begin{aligned}
\mathcal{F}_{A B} & =\frac{1}{24 !} F_{\text {Iabcd }}^{(5)}\left(C_{10} \Gamma_{(10)}^{I a b c d}\right)_{A B} \dot{\dot{\alpha}}+\sum_{n=1,3,5} \frac{1}{2 n !} F_{I_{1} . . I_{n}}^{(n)}\left(C_{10} \Gamma_{(10)}^{I_{1} . . I_{n}}\right)_{A B} \dot{\alpha} \dot{\alpha} \\
& =F_{I 5678}^{(5)}\left(C \Gamma^{I}\right)_{A B}+\sum_{n=1,3,5} \frac{1}{n !} F_{I_{1} . . I_{n}}^{(n)}\left(C \Gamma^{I_{1} . . I_{n}}\right)_{A B},
\end{aligned}
$$


where, in the last line, we have used $\Gamma_{(10)}^{5678}=-\Gamma_{(10)}^{33 \overline{3} \overline{4}}=1_{(6)} \otimes\left(-\gamma^{\mathrm{ND}}\right)$ and that $-\gamma^{\mathrm{ND}}$ is 1 on the indices $\dot{\alpha}$ (see (3.4)). Lorentz invariance again fixes the form of $\mathcal{A}_{R}$ up to a constant

$$
\mathcal{A}_{\mathrm{R}}=2 \mathcal{I} \bar{\mu}^{A}\left(C^{-1} \mathcal{F} R C^{-1}\right)_{A B} \mu^{B},
$$

where the factor of two in evidence comes from the trace over the $4 \mathrm{D}$ spinor indices. Notice that both the D1 and the D5 reflections matrices (3.17) reduce in 6D to the expression $R=\Gamma^{t y}$, since they differs by $\Gamma_{(10)}^{5678}$ which is just the identity on the spinor components $(\dot{\alpha})$ entering in this computation. Due to the symmetry properties of the open string condensate, this result is non-vanishing only when the Gamma matrices in $\mathcal{F}$ and $R$ reconstruct a 3 -form (3.11). This is possible for all terms in (4.22), thus 1, 3 and 5 -forms components of the RR field will all contribute to the string amplitude. We use $\mathcal{A}_{\mathrm{R}}[n]$ to indicate the contribution of the form of degree $n$. By using again the second relation in (3.11), we get

$$
\begin{aligned}
& \mathcal{A}_{\mathrm{R}}[1]=16 \mathcal{I} v_{t y i} F_{i}^{(1)}, \\
& \mathcal{A}_{\mathrm{R}}[3]=16 \mathcal{I}\left(\frac{1}{2} v_{y i j} F_{t i j}^{(3)}-\frac{1}{2} v_{t i j} F_{y i j}^{(3)}\right), \\
& \mathcal{A}_{\mathrm{R}}[5]=-16 \mathcal{I}\left(\frac{1}{3 !} v_{i j k} F_{t y i j k}^{(5)}-v_{t y i} F_{i 5678}^{(5)}\right) .
\end{aligned}
$$

To evaluate $\mathcal{I}$, one can take a specific choice of the open and closed string polarizations; for instance, it is convenient to choose for $\bar{\mu}^{A}, \mu^{B}$ the following weights $\vec{\epsilon}_{A}=\vec{\epsilon}_{B}=(---)$. The relevant correlators are

$$
\begin{aligned}
& \left\langle\Delta\left(z_{1}\right) \Delta\left(z_{4}\right)\right\rangle=z_{14}^{-\frac{1}{2}}, \quad\left\langle S^{\dot{\alpha}}\left(z_{2}\right) S^{\dot{\beta}}\left(z_{3}\right)\right\rangle=z_{23}^{-\frac{1}{2}} \epsilon^{\dot{\alpha} \dot{\beta}}, \quad\left\langle\prod_{i} e^{-\frac{\varphi}{2}}\left(z_{i}\right)\right\rangle=\prod_{i<j} z_{i j}^{-\frac{1}{4}} \\
& \left\langle S^{---}\left(z_{1}\right) S^{+++}\left(z_{2}\right) S^{+++}\left(z_{3}\right) S^{---}\left(z_{4}\right)\right\rangle=\left(\frac{z_{14} z_{23}}{z_{12} z_{13} z_{24} z_{34}}\right)^{\frac{3}{4}}
\end{aligned}
$$

Assembling the various correlators together, and using again the measure (4.2), one finds

$$
\mathcal{I}=\frac{1}{4 \sqrt{2}} \int \frac{d \omega}{\omega}=\frac{\pi \mathrm{i}}{2 \sqrt{2}} .
$$

We can now show that the amplitude (4.24) contains the $1 / r^{3}$ contributions to the RR fields in the solution (2.2) that are characterized by the functions $A_{i}, B_{i}, \mathcal{A}$ and $\mathcal{B}$. We first need to rewrite the result (4.24) in terms of the gauge potentials $C^{(n-1)}$ by using

$$
F_{I_{1} . . I_{n}}^{(n)}=n \mathrm{i} k_{\left[I_{1}\right.} C_{\left.I_{2} . . I_{n}\right]}^{(n-1)} .
$$

So we get

$$
\begin{aligned}
\mathcal{A}_{\mathrm{R}}[1] & =-4 \sqrt{2} \pi k_{i} C^{(0)} v_{t y i}, \\
\mathcal{A}_{\mathrm{R}}[3] & =-4 \sqrt{2} \pi\left(\frac{1}{2} k_{j} C_{t i}^{(2)} v_{y i j}-\frac{1}{2} k_{j} C_{y i}^{(2)} v_{t i j}\right) \\
& =+4 \sqrt{2} \pi\left(\frac{1}{4} k_{j} C^{(2) t i} \epsilon_{i j k l} v_{t k l}+\frac{1}{2} k_{j} C^{(2) y i} v_{t i j}\right), \\
\mathcal{A}_{\mathrm{R}}[5] & =4 \sqrt{2} \pi\left(\frac{1}{2} k^{i} C_{t y j k}^{(4)} v_{i j k}-k^{i} C_{5678}^{(4)} v_{t y i}\right) \\
& =4 \sqrt{2} \pi\left(\frac{1}{2} \epsilon_{i j k l} k^{i} C^{(4) t y j k} v_{t y l}-k^{i} C^{(4) 5678} v_{t y i}\right) .
\end{aligned}
$$


We then extract from the amplitude the gauge field profile

$$
C_{\mu_{1} \ldots \mu_{n}}^{(n)}(k)=\frac{\delta \mathcal{A}_{\mathrm{R}}}{\delta C^{(n) \mu_{1} \ldots \mu_{n}}} \quad\left(\mu_{1}<\mu_{2} \ldots<\mu_{n}\right),
$$

attach a free propagator to each profile and take the Fourier transform, as explained in (4.14). Hence (4.28) yields the following results for the large distance behavior of the RR fields

$$
\begin{aligned}
C^{(0)}(x) & =\frac{2 \sqrt{2}}{\pi} \frac{x_{i} v_{t y i}}{r^{4}}, \\
C_{t i}^{(2)}(x) & =-\frac{1}{\sqrt{2} \pi} \epsilon_{i j k l} \frac{x_{j} v_{t k l}}{r^{4}}, \quad C_{y i}^{(2)}(x)=-\frac{\sqrt{2}}{\pi} \frac{x_{j} v_{t i j}}{r^{4}}, \\
C_{t y i j}^{(4)}(x) & =-\frac{2 \sqrt{2}}{\pi} \epsilon_{i j k l} \frac{x_{k} v_{t y l}}{r^{4}}, \quad C_{5678}^{(4)}(x)=\frac{2 \sqrt{2}}{\pi} \frac{x_{i} v_{t y i}}{r^{4}} .
\end{aligned}
$$

Using the identifications (4.19), the values above exactly reproduce the supergravity result (2.13).

\section{Conclusions}

We have shown how the asymptotic expansion of the 2-charge fuzzball geometries (2.2) is reproduced by computing string amplitudes for the emission of a closed string state from a disk with mixed D1D5 boundary conditions. Each fuzzball geometry is completely determined by a curve $f_{A}(v)$ that captures how different the solution is from the naive D1D5 superposition. Microscopically the information about the curve $f_{A}(v)$ is encoded in a condensate for the open strings stretched between the two types of D-branes. In order to derive the exact dictionary between $f_{A}(v)$ and the string condensate one should compute the closed string emission from a disk in presence of a finite value for the open condensate. This is a challenging task since the open string states stretched between the D1 and the D5 branes contain twist fields. However we could explicitly check this dictionary by treating the open condensates perturbatively: in the large distance limit the $1 / r^{3}$ terms of the fuzzball solutions match the gravitational backreaction of the D-brane system when the open string condensates are included at first order. We believe that a similar pattern exists also at higher orders and that it is possible to construct the dictionary term by term in the perturbative expansion without changing the identifications established at lower orders.

Our analysis is not complete: in this paper we have reproduced only a subset of the D1D5 geometries, those that are invariant under the $\mathrm{SO}(4)$ rotations of the compact space $T^{4}$ (in the notation of section 2 these are the geometries with $\mathcal{A}_{\hat{a}}=0$ ). These geometries are dual to those Ramond ground states of the D1D5 CFT that are associated with the "universal sub-sector" of the cohomology of $T^{4}$ (i.e. the $(0,0),(2,2),(2,0),(0,2)$ forms, and the Kähler $(1,1)$ form). Hence the geometries we consider do not use in any way the properties of the compact space $T^{4}$ and the results of our computation apply, with no modification, to the case in which the compactification manifold is K3. We have left open the problem of identifying which open string condensates generate the geometries associated with the remaining even cohomology of $T^{4}$. We expect these latter configurations to be 
related to the ones we have considered here by the action of the supersymmetries broken by the D1 and D5 branes. A similar approach was used in [32] to study spin potentials of $\frac{1}{2}$-BPS Dp-branes. Moreover, in the case of $T^{4}$, one also has the microstates associated with the odd cohomology, which correspond, in the duality frame of the fundamental string, to fundamental string states with pairs of fermionic excitations [11]. One can show that these states are distinct form the ones we have considered here: indeed, all the geometries in the class of [11] display $1 / r^{3}$ corrections in the $g_{t t}$ and $g_{y y}$ metric components, which are absent for the states we identify here. There must exist open string condensates generating also those geometries, and it is an interesting problem to find them.

From a more general point of view, we think that our computation provides an important relation between the gravitational description of the D1D5 microstates and their microscopic description in terms of D-branes. Most of the studies of the fuzzball solutions focused on the near-"horizon" geometry and its relation with the dual CFT description. However the full microstate geometry is asymptotically flat and the large $r$ limit represents a regime where both the gravitational and the D-brane descriptions are valid. Clearly, in the string amplitude computation, we are able to explore only perturbatively the Higgs branch, by inserting a finite number of twist vertex operators associated to the strings stretched between the D1 and the D5 branes. However, this is sufficient to capture some of the distinctive properties of the microstate geometries and provides a direct support to the idea that the D-brane configurations used to compute the entropy at $g_{s}=0$ evolve into fuzzballs when the string coupling is turned on.

An advantage of our approach is that it is completely systematic and allows, in principle, to investigate a large number of open problems. In our opinion, two of the most important open issues are the study of string corrections and the construction of the geometry dual to a general 3-charge microstate. With our approach one could derive the asymptotic expansion for such a general 3-charge geometry, and this could provide an important clue for constructing the full exact solution. Within our framework, one could also obtain the higher order string corrections to the microstate geometries without the need of guessing what term in the gravitational effective action are the relevant one in the different configuration. We hope to come back to these issues in a subsequent work.

\section{Acknowledgments}

We would like to thank I. Bena, S. Mathur and C. Ruef for discussions. This work is partially supported by the ERC Advanced Grant no. 226455, "Supersymmetry, Quantum Gravity and Gauge Fields" (SUPERFIELDS) and by STFC under the Rolling Grant $\mathrm{ST} / \mathrm{G} 000565 / 1$.

Open Access. This article is distributed under the terms of the Creative Commons Attribution Noncommercial License which permits any noncommercial use, distribution, and reproduction in any medium, provided the original author(s) and source are credited. 


\section{References}

[1] A. Sen, Extremal black holes and elementary string states, Mod. Phys. Lett. A 10 (1995) 2081 [hep-th/9504147] [SPIRES].

[2] A. Strominger and C. Vafa, Microscopic Origin of the Bekenstein-Hawking Entropy, Phys. Lett. B 379 (1996) 99 [hep-th/9601029] [SPIRES].

[3] S.D. Mathur, The fuzzball proposal for black holes: An elementary review, Fortsch. Phys. 53 (2005) 793 [hep-th/0502050] [SPIRES].

[4] I. Bena and N.P. Warner, Black holes, black rings and their microstates, Lect. Notes Phys. 755 (2008) 1 [hep-th/0701216] [SPIRES].

[5] K. Skenderis and M. Taylor, The fuzzball proposal for black holes, Phys. Rept. 467 (2008) 117 [arXiv:0804.0552] [SPIRES].

[6] V. Balasubramanian, J. de Boer, S. El-Showk and I. Messamah, Black Holes as Effective Geometries, Class. Quant. Grav. 25 (2008) 214004 [arXiv:0811.0263] [SPIRES].

[7] O. Lunin and S.D. Mathur, Metric of the multiply wound rotating string, Nucl. Phys. B 610 (2001) 49 [hep-th/0105136] [SPIRES].

[8] O. Lunin and S.D. Mathur, AdS/CFT duality and the black hole information paradox, Nucl. Phys. B 623 (2002) 342 [hep-th/0109154] [SPIRES].

[9] O. Lunin, S.D. Mathur and A. Saxena, What is the gravity dual of a chiral primary?, Nucl. Phys. B 655 (2003) 185 [hep-th/0211292] [SPIRES].

[10] O. Lunin, J.M. Maldacena and L. Maoz, Gravity solutions for the D1 - D5 system with angular momentum, hep-th/0212210 [SPIRES].

[11] M. Taylor, General 2 charge geometries, JHEP 03 (2006) 009 [hep-th/0507223] [SPIRES].

[12] I. Kanitscheider, K. Skenderis and M. Taylor, Fuzzballs with internal excitations, JHEP 06 (2007) 056 [arXiv:0704.0690] [SPIRES].

[13] O. Lunin, Adding momentum to D1 - D5 system, JHEP 04 (2004) 054 [hep-th/0404006] [SPIRES].

[14] S. Giusto, S.D. Mathur and A. Saxena, Dual geometries for a set of 3-charge microstates, Nucl. Phys. B 701 (2004) 357 [hep-th/0405017] [SPIRES].

[15] S. Giusto and S.D. Mathur, Geometry of D1 - D5 - P bound states, Nucl. Phys. B 729 (2005) 203 [hep-th/0409067] [SPIRES].

[16] I. Bena and N.P. Warner, Bubbling supertubes and foaming black holes, Phys. Rev. D 74 (2006) 066001 [hep-th/0505166] [SPIRES].

[17] P. Berglund, E.G. Gimon and T.S. Levi, Supergravity microstates for BPS black holes and black rings, JHEP 06 (2006) 007 [hep-th/0505167] [SPIRES].

[18] I. Bena, C.-W. Wang and N.P. Warner, Mergers and Typical Black Hole Microstates, JHEP 11 (2006) 042 [hep-th/0608217] [SPIRES].

[19] I. Bena, N. Bobev, C. Ruef and N.P. Warner, Entropy Enhancement and Black Hole Microstates, arXiv:0804.4487 [SPIRES].

[20] P. Di Vecchia et al., Classical p-branes from boundary state, Nucl. Phys. B 507 (1997) 259 [hep-th/9707068] [SPIRES]. 
[21] M. Bertolini et al., Is a classical description of stable non-BPS D-branes possible?, Nucl. Phys. B 590 (2000) 471 [hep-th/0007097] [SPIRES].

[22] A. Dabholkar, J.P. Gauntlett, J.A. Harvey and D. Waldram, Strings as Solitons and Black Holes as Strings, Nucl. Phys. B 474 (1996) 85 [hep-th/9511053] [SPIRES].

[23] C.G. Callan, J.M. Maldacena and A.W. Peet, Extremal Black Holes As Fundamental Strings, Nucl. Phys. B 475 (1996) 645 [hep-th/9510134] [SPIRES].

[24] D. Martelli and J.F. Morales, Bubbling AdS $S_{3}$, JHEP 02 (2005) 048 [hep-th/0412136] [SPIRES].

[25] C. Bachas, Relativistic string in a pulse, Ann. Phys. 305 (2003) 286 [hep-th/0212217] [SPIRES].

[26] Y. Hikida, H. Takayanagi and T. Takayanagi, Boundary states for D-branes with traveling waves, JHEP 04 (2003) 032 [hep-th/0303214] [SPIRES].

[27] M. Billó et al., Classical gauge instantons from open strings, JHEP 02 (2003) 045 [hep-th/0211250] [SPIRES].

[28] M. Billó' et al., Flux interactions on D-branes and instantons, JHEP 10 (2008) 112 [arXiv: 0807.1666] [SPIRES].

[29] J. Polchinski, String theory. Vol. 2: Superstring theory and beyond, Cambridge University Press, Cambridge U.K. (1998).

[30] M. Billó et al., Microscopic string analysis of the D0-D8 brane system and dual RR states, Nucl. Phys. B 526 (1998) 199 [hep-th/9802088] [SPIRES].

[31] V.A. Kostelecky, O. Lechtenfeld, W. Lerche, S. Samuel and S. Watamura, Conformal Techniques, Bosonization and Tree Level String Amplitudes, Nucl. Phys. B 288 (1987) 173 [SPIRES].

[32] J.F. Morales, C.A. Scrucca and M. Serone, Scale independent spin effects in D-brane dynamics, Nucl. Phys. B 534 (1998) 223 [hep-th/9801183] [SPIRES]. 